\title{
Genetic analysis of parathyroid and pancreatic tumors in a patient with multiple endocrine neoplasia type 1 using whole-exome sequencing
}

Bo-Young Kim ${ }^{1+}$, Mi-Hyun Park ${ }^{1 \dagger}$, Hae-Mi Woo ${ }^{1}$, Hye-Yeong Jo ${ }^{1}$, Ji Hoon Kim³ ${ }^{3}$ Hyung Jin Choi ${ }^{2 *}$ and Soo Kyung Koo ${ }^{1 *}$ (iD

\begin{abstract}
Background: Multiple endocrine neoplasia type 1 (MEN1) syndrome is an autosomal dominant hereditary disorder characterized by the presence of endocrine tumors affecting the parathyroid, pancreas, and pituitary. A heterozygous germline inactivating mutation in the MEN1 gene (first hit) may be followed by somatic loss of the remaining normal copy or somatic mutations in the MEN1 gene (second hit). Whole-exome sequencing has been successfully used to elucidate the mutations associated with the different types of tumors.

Case presentation: We performed whole-exome sequencing (WES) on three parathyroid tumors, one pancreatic insulinoma, and a blood sample taken from the same patient with MEN1 to study tumor heterogeneity in MEN1 originating from different tumors. We identified a novel frame-shift deletion (c.1382_1383delAG, p.E461GfsX69) in the MEN1 gene using WES, which was confirmed by Sanger sequencing. WES and the SNP array revealed somatic LOH on chromosome 11 in parathyroid tumors (left upper, left lower, and right upper parathyroid). However, we did not detect a somatic MEN1 gene mutation or LOH in the pancreatic insulinoma. WES revealed two somatic functional variants outside the MEN1 gene in the pancreatic insulinoma.

Conclusions: This study revealed heterogeneity among tumors in the same patient with MEN1, suggesting that different tumor-specific tumorigenic mechanisms may contribute to the pathogenesis of MEN1 tumors. The present study supports the clinical applicability of the WES strategy to research on multiple tumor samples and blood.
\end{abstract}

Keywords: Multiple endocrine neoplasia type 1, Genetic analysis, Somatic mutation, Whole-exome sequencing, Clinical genomics, Case report

\section{Background}

Multiple endocrine neoplasia type 1 (MEN1) is an autosomal dominant inherited tumor syndrome characterized by the combined tumors of the parathyroid glands, pancreas, and pituitary gland [1]. Parathyroid tumors are the most common clinical manifestation, and the

\footnotetext{
* Correspondence: hjchoi@snu.ac.kr; skkoo@nih.go.kr

${ }^{\dagger}$ Equal contributors

${ }^{2}$ Department of Anatomy, Department of Biomedical Science, Neuroscience Research Institute, Seoul National University College of Medicine, 28 Yongon-dong, Chongno-gu, Seoul, South Korea

'Division of Intractable Diseases, Center for Biomedical Sciences, Korea National Institute of Health, 187 Osongsaengmyeing2-ro, Cheongju-si, Chungcheongbuk-do 28159, South Korea

Full list of author information is available at the end of the article
}

prevalence of hyperparathyroidism is $>90 \%$ in patients with MEN1, whereas those of pancreatic and pituitary tumors are $40-70 \%$ and $30-60 \%$, respectively [2]. MEN1 is caused by germline mutations in the MEN1 tumor suppressor gene linked to chromosomal locus 11q13 [3]. A heterozygous germline-inactivating mutation of the MEN1 gene (first hit) may be followed by loss of the normal copy of this gene or a somatic inactivating mutation (second hit), leading to complete loss of function of the encoded protein menin $[4,5]$. A MEN1 gene mutation analysis has helped identify the cause of various sporadically occurring tumors of the same type that are observed in patients with MEN1 syndrome. 
Identifying the genetic cause of the disease has become useful for diagnosing at-risk carriers affected by MEN1, and whole-exome sequencing (WES) approaches could help identify the causative genes [6]. Such an approach has been successfully used to elucidate mutations associated with multiple tumor types. Since next-generation sequencing (NGS) technology was introduced in 2008 [7], comprehensive genomic analyses have accelerated. With the power to detect novel variants from only a small number of individuals, NGS is proving invaluable for modern geneticists, boasting putative diagnostic rates of $21-25 \%$ for rare diseases of unknown etiology [8, 9]. Therefore, we pursued a WES analysis of parathyroid adenomas and a pancreatic insulinoma to study the tumor heterogeneity that may be contributing to different mechanisms of tumor-specific tumorigenesis.

\section{Case presentation}

The proband patient was admitted to hospital with a chief complaint of flank pain. The patient had been sweating for 5 years, and was nervous when fasting. The patient had had multiple episodes of kidney stones during the past 2 years. Table 1 presents the patient's clinical information. The serum calcium level was $13.7 \mathrm{mg} / \mathrm{dL}$ (reference range, 8.2-10.8 $\mathrm{mg} / \mathrm{dL}$ ), and serum intact PTH was $209 \mathrm{pg} / \mathrm{mL}$ (reference range, 6-8 pg/mL), indicating primary hyperparathyroidism. The fasting serum glucose was $48 \mathrm{mg} / \mathrm{dL}$ (reference range, $70-110 \mathrm{mg} / \mathrm{dL}$ ), and fasting serum insulin was $21.9 \mu \mathrm{U} / \mathrm{mL}$, with a serum C-peptide level of $3.00 \mathrm{ng} / \mathrm{mL}$ (reference range, 1.06-3.53 $\mathrm{ng} / \mathrm{mL}$ ), indicating endogenous hyperinsulinemia. The anterior pituitary hormones were within the normal ranges. Sestamibi parathyroid, neck computed tomography (CT), and neck ultrasonography revealed multiple parathyroid adenomas. Abdominal CT revealed an approximately $1.6-\mathrm{cm}$ well-defined

Table 1 Clinical details of the patient with multiple endocrine neoplasia type 1

\begin{tabular}{|c|c|c|c|c|}
\hline \multicolumn{4}{|l|}{ Characteristic } & \multirow[t]{2}{*}{ Reference } \\
\hline Sex/Age (yr) & $M / 33$ & & & \\
\hline \multicolumn{5}{|c|}{ Primary hyperparathyroidism (Parathyroid adenoma) } \\
\hline Calcium & 13.7 & $\Delta$ & $\mathrm{mg} / \mathrm{dL}$ & $8.2-10.8$ \\
\hline Phosphorus & 2.3 & $\nabla$ & $\mathrm{mg} / \mathrm{dL}$ & $2.5-5.5$ \\
\hline PTH-intact & 209 & $\Delta$ & $\mathrm{pg} / \mathrm{mL}$ & $8-6$ \\
\hline PTH related peptide (PTHrP) & 1.1 & & & $<1.1$ \\
\hline Creatinine & 2.00 & $\Delta$ & $\mathrm{mg} / \mathrm{dL}$ & $0.60-0.20$ \\
\hline \multicolumn{5}{|c|}{ Insulinoma (enteropancreatic tumor) } \\
\hline C-peptide (serum) & 3.00 & & $\mathrm{ng} / \mathrm{mL}$ & $1.06-3.53$ \\
\hline Glucose & 48 & $\nabla$ & $\mathrm{mg} / \mathrm{dL}$ & $70-110$ \\
\hline Insulin & 21.9 & $\boldsymbol{\Delta}$ & $\mu \mathrm{UU} / \mathrm{mL}$ & \\
\hline
\end{tabular}

enhancing mass at the head of the pancreas, suggesting an endocrine tumor. Brain sellar magnetic resonance imaging showed a normal pituitary. A subtotal parathyroidectomy and pancreaticoduodenectomy were performed. Postoperatively, all hormones and serum calcium returned to their normal ranges (Fig. 1).

\section{Whole-exome sequencing and validation with Sanger sequencing}

Genomic DNA was isolated from matched tumor tissues and a constitutional blood sample using standard protocols included in the commercially available QIAGEN DNeasy Blood and Tissue kit (QIAGEN, Hilden, Germany). All specimens were quality control checked for purity using a NanoDrop spectrophotometer and Qubit Fluorometer (Thermo Fisher Scientific Inc., Waltham, MA, USA). Tumor and blood DNA were captured on the Agilent SureSelect DNA library construction (SureSelect V4 + UTR; Agilent Technologies, Palo Alto, CA, USA) and were sequenced on the Illumina Hiseq2000 instrument (Illumina, Inc., San Diego, CA, USA), as described by the manufacturer's protocols for paired 101-bp reads. Image analysis and base calling was performed using Illumina pipeline ver. 2.3 with default parameters.

Reads were aligned to the human reference hg19 genome assembly using the Burrows-Wheeler Aligner (BWA; http://bio-bwa.sourceforge.net/), and duplicated read pairs were removed [10]. Genotypes for single nucleotide variants (SNVs) and short indels were called using the Genome Analysis Toolkit (GATK) [11] and Sequence Alignment Map tool (SAMtools, http://samtools.sourceforge.net/), respectively. Variants were annotated using ANNOVAR (http://www.openbioinformatics.org/annovar/). Allele frequencies for each variant were estimated based on phase 1 genotypes from the 1000 Genomics Project, the Korean control exome dataset, which includes the Korean Reference Genome (KRG database, $n=622$ ). The effects of the identified variants were assessed using the Sorting Intolerant Form Tolerant (SIFT, http://sift.jcvi.org), and PolyPhen-2 (http://genetics.bwh.harvard.edu/pph2), automatic tools for predicting the possible impact of an amino acid substitution on the structure and function of a human protein $[12,13]$.

Sanger sequencing was used as an alternate platform to validate the identified variants in the four exome-sequenced tumor samples. Primer3 (http:// frodo.wi.mit.edu/primer3/) was used to generate primers to amplify variants identified via exome sequencing. Amplicons from blood and tumor genomic DNA were analyzed using gel electrophoresis and were sequenced using an ABI 3730 genetic analyzer (Applied Biosystems, Foster City, CA) with forward and reverse primers. 


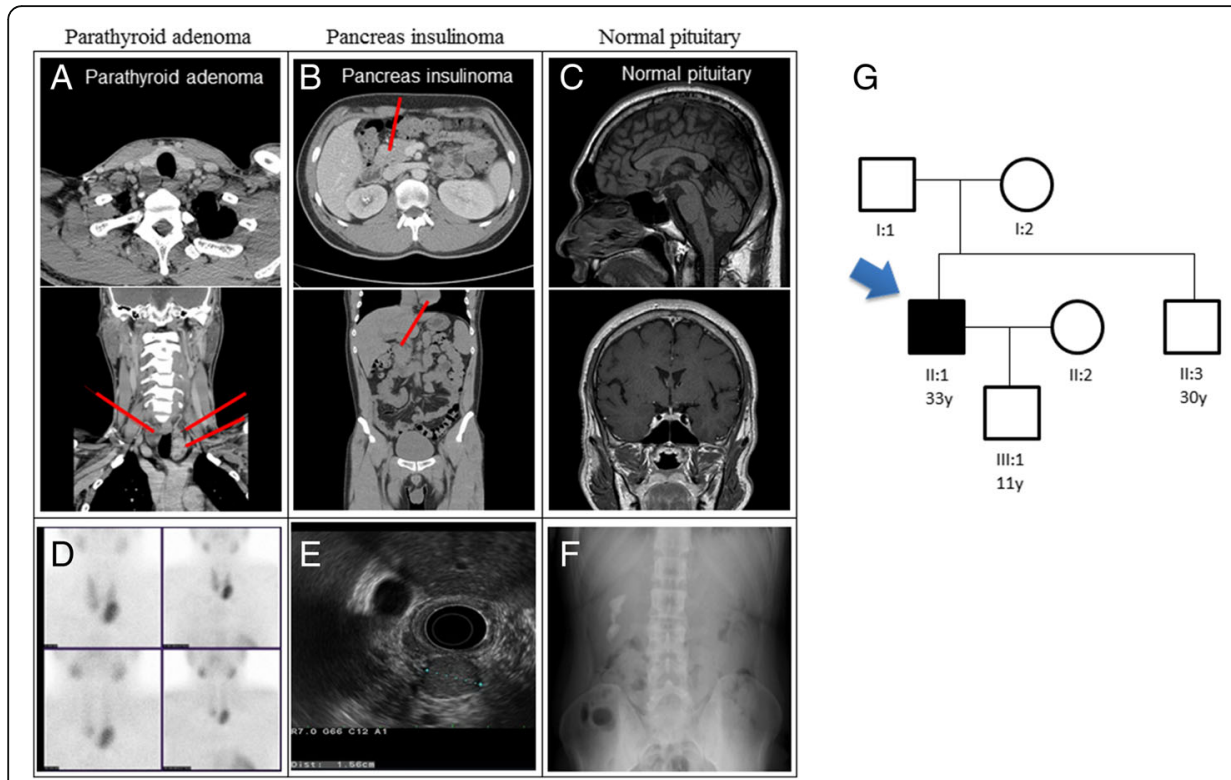

$\mathrm{H}$

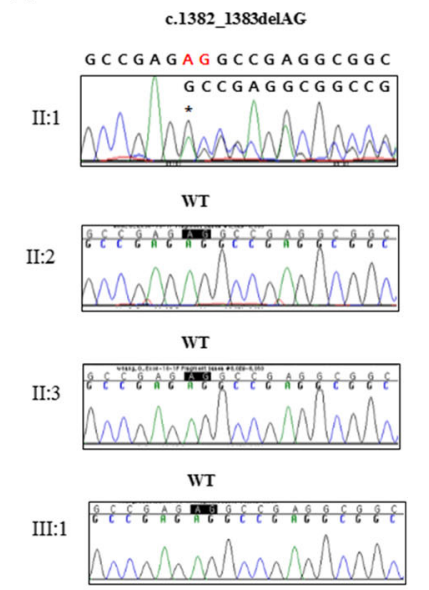

Fig. 1 Case subject. a Neck computed tomography (CT) scan: Heterogeneous enhancing mass with cystic changes in both paratracheal (right $1.5 \mathrm{~cm}$, left $2.2 \mathrm{~cm}$ ) and parathyroid adenomas. $\mathbf{b}$ Abdominal $C T$ : About a 1.6- $\mathrm{cm}$ well-defined enhancing mass at the head of the pancreas; R/O endocrine tumor. c Sellar magnetic resonance imaging: Normal pituitary. d Sestamibi parathyroid scan: Multiple parathyroid adenoma. e Endoscopic ultrasound and fine need aspiration: About a 1.6-cm-sized round hypoechoic homogenous mass at the pancreatic head. Immunohistochemistry: Ki-67 index $<1 \%$, P53: (-), synaptophysin: (+), neuroendocrine tumor. $\mathbf{f}$ KUB X-ray: Huge calyceal stones, Rt. kidney. $\mathbf{g}$ Pedigree of the patient with multiple endocrine neoplasia type1 (MEN1). The affected subject is indicated by the arrow. $\mathbf{h}$ Novel germline mutation in the MEN1 gene. Sanger sequence of MEN1 codons 462 in a wild-type subject and patient are shown and confirmed the c.1382_1383delAG mutation. Mutated residue is indicated by an asterisk, and the encoded amino acids are shown as single-letter code

\section{Genotyping and identification of loss of heterozygosity}

Samples were genotyped with the Illumina Human Omni2.5-8 BeadChip according to the manufacturer's protocols, and the genotypes were called using GenomeStudio software (Illumina) with standard cluster definitions. GenomeStudio provides raw data normalization, clustering, and genotype calling and calculates a GenCall score for each genotype, which is a measure of the accuracy of the genotype call. A 1-Mb sliding window across each chromosome was used to determine large regions with $\mathrm{LOH}$ in the tumor samples. The SNP array extracted both the $\log _{2}$ intensity ratio $(\operatorname{LogR})$ and the $B$ allele frequency (BAF) data. LogR measures the intensity of a sample relative to the reference intensity, and BAF measures the allele frequency at a particular SNP along the genome.

\section{Whole-exome sequence analysis}

We performed WES on three parathyroid and one pancreatic tumor specimens as well as on a whole blood sample from the same patient diagnosed with MEN1. We sequenced the tumor and germline DNA isolated from whole blood to identify somatic changes in the tumors.

We provided an analytical pipeline to detect germline or tumor-specific somatic variations in which each sample was considered independently. The genomic pipeline is outlined in Fig. 2a. Raw reads (fastq files) were first quality checked with FastQC. Filtered reads were aligned to the appropriate reference genome using BWA, and duplicate reads were marked using Picard. As the tumor and blood BAM files were merged to detect the somatic variants, the SNVs and indel calling were performed using two separate callers to assist in identifying truepositive somatic variant calls while reducing the falsepositive calls.

WES metrics and summary statistics for each specimen are shown in Fig. 2b. We generated nearly 100 billion total reads from WES, for an average mapped coverage of 66-98. To evaluate the overall quality of the variant data, germline SNVs were called, and the transition to transversion and $135 \mathrm{dbSNP}$ (Single Nucleotide Polymorphism Database) concordance ratios were calculated. We used the GATK and SAMtools programs to identify somatic SNVs and indels, respectively, and found a high validation rate for the variants called by program. The identified variants were combined into single file and annotated using ANNOVAR to add information, such the gene variants, consequence of the mutation (nonsynonymous, nonsense, etc.), and information from databases (e.g., PolyPhen2, SIFT, dbSNP, OMIN, and COSMIC). The final output of the pipeline consisted of a single file containing the annotated variants 


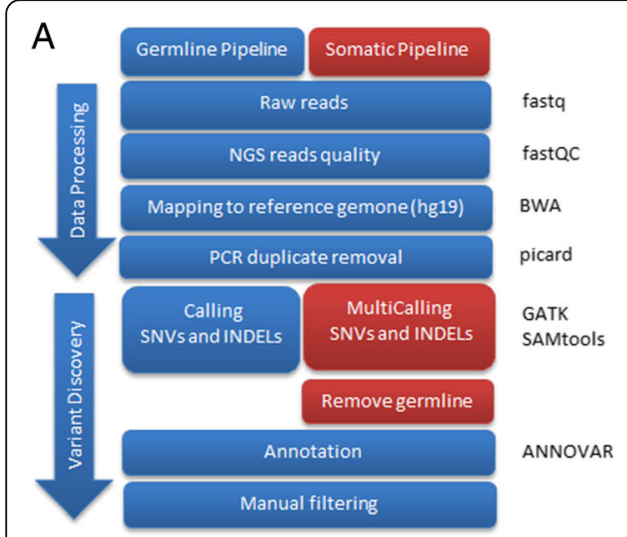

\begin{tabular}{|c|c|c|c|c|c|}
\hline Parameter & $\begin{array}{l}\text { Parathyroid } \\
\text { Left Upper }\end{array}$ & $\begin{array}{l}\text { Parathyroid } \\
\text { Left Lower }\end{array}$ & $\begin{array}{l}\text { Parathyroid } \\
\text { Right Upper }\end{array}$ & Pancreas & Blood \\
\hline Total yield (bp) & $12,478,044,394$ & $8,824,825,106$ & $9,917,236,662$ & $10,406,905,266$ & $9,511,127,176$ \\
\hline Total reads & $123,544,994$ & $87,374,506$ & $98,190,462$ & $103,038,666$ & $94,169,576$ \\
\hline On-target reads & $66,749,329$ & $66,598,185$ & $53,169,178$ & $77,879,066$ & $58,883,647$ \\
\hline$\%$ On-target reads & $89.10 \%$ & $\$ 6.30 \%$ & $87.60 \%$ & $88.00 \%$ & $89.70 \%$ \\
\hline Coverage of target regions & 85 & 85 & 66 & 98 & 76 \\
\hline \multicolumn{6}{|l|}{ GATK } \\
\hline Total variants & 113,501 & 117,059 & 107,007 & 118,195 & 114,724 \\
\hline Filtering blood & 5,338 & 5,567 & 4,833 & 5,459 & \\
\hline Exonic variants & 334 & 325 & 291 & 340 & \\
\hline Somatic SNVs called & 327 & 319 & 285 & 333 & \\
\hline Somatic INDELs called & 7 & 6 & 6 & 7 & \\
\hline \multicolumn{6}{|l|}{ SAMtools } \\
\hline Total variants & 74,095 & 79,314 & 66,872 & 79,710 & 70,727 \\
\hline Filtering Blood & 2,239 & 1,570 & 3,080 & 1,773 & \\
\hline Exonic variants & 577 & 317 & 916 & 341 & \\
\hline Somatic SNVs called & 537 & 273 & 842 & 283 & \\
\hline Somatic INDELs called & 39 & 44 & 74 & 58 & \\
\hline
\end{tabular}

Fig. 2 Whole-exome sequencing analytical pipelines. a Two workflows are present: Germline detection and somatic detection. Red highlights the difference between our germline pipeline and the somatic pipeline. b Summary exome sequencing statistics for all four tumor tissues and blood

from the tumor and blood samples. These analyses indicated that no biases were encountered with respect to nucleotide substitutions. SNPs identified in the data were strongly correlated with common genetic variations, indicating that high-quality variant calling was performed.

\section{Novel germline mutation in the MEN1 gene}

The nine exons of the MEN1 gene as well as their flanking introns were amplified and subjected to Sanger sequencing. Herein, we confirmed four germline variants of the MEN1 gene by WES and Sanger sequencing, and the distribution is shown in Table 2. Mutations were found in exons 9 and 10 (one frameshift deletion, one missense mutation, and two synonymous variants). A novel germline mutation (c.1382_1383delAG/p.E462GfsX69) was newly confirmed in the present study, and the other three have been reported previously in the 1000 Genome Project and the Korean control exome dataset. This novel germline mutation (c.1382_1383delAG) was also present in all four tumor samples according to the WES analysis. The brother and son of the proband did not harbor this mutation (Fig. 1h). All variants were confirmed based on the NCBI SNP database (http://www.cnbi.nlm.nih.gov/ SNP) and the Human Gene Mutation Database (http:// www.hgmd.org).

\section{Detecting loss of heterozygosity}

Oligonucleotide-based SNP genotyping array has been used to measure physical copy number and genetic aberrations, such as $\mathrm{LOH}$. We included three additional MEN1 parathyroid specimens and one pancreatic specimen for which there was a paired germline sample (blood). Three tissue samples (left upper, left lower, and right upper parathyroid) had chromosomal allelic imbalance events (LOH with chromosomal loss).

Additional file 2: Figure S1 illustrates a greater degree of chromosomal allelic imbalance across the MEN1 tumor genomes compared with that in the blood sample. The SNP array collects intensity and allelic information, allowing two different genomic profiles to be generated. The $\log 2$ intensity ratio measures the intensity of a sample relative to a reference intensity, and the $B$ allele frequency (BAF) measures the allele frequency at a particular SNP along the genome. The expected BAF value at a normal (non-LOH) polymorphic position is 0.5 , and a significant deviation from 0.5 identifies $\mathrm{LOH}$. LOH can easily be observed using the BAF plot by noting an absence of heterozygotes. Of note, there was consistent LOH of chromosome 11 (where the MEN1 gene resides) across all three of the parathyroid tumor samples from the patient with MEN1, whereas the pancreatic tumor exhibited no chromosomal $\mathrm{LOH}$ events

\section{Identifying tumor-specific somatic mutations}

WES was performed on tumor and constitutional DNA samples from the patient with MEN1. The raw sequencing data were mapped to the hg19 reference human genome, the variants were identified, and tumor and constitutional blood sequences from each tumor were cross-referenced to identify somatic variants unique to each tumor genome.

WES was performed on the three parathyroid and one pancreatic tumors demonstrated an average of 180 variants in GATK and 389 in SAMtools. Additional file 1: Table S1 summarizes the number of somatic variants observed in the parathyroid and pancreatic tumors. Total somatic variants were examined using computational metrics and manual read alignment analysis to 


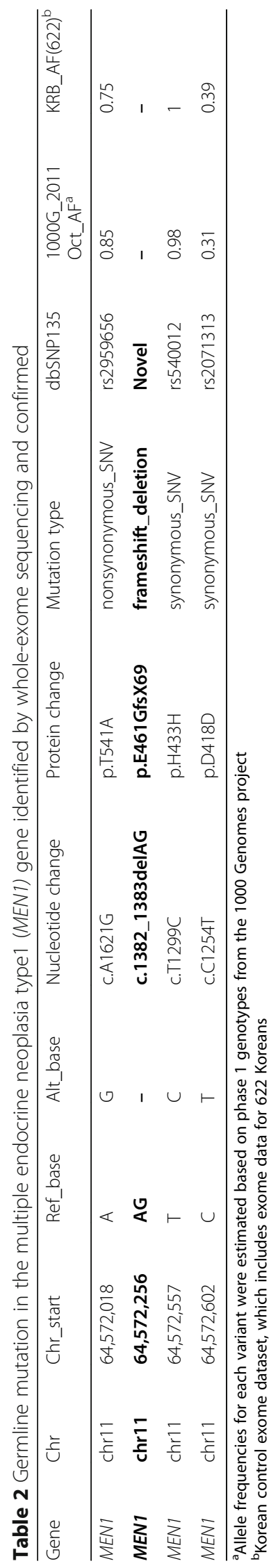


identify mutations with a high likelihood of being false positives.

The variants identified were annotated based on novelty, impact on the encoded protein, and conservation. Across all four tumor types, nine variants were identified in the coding region; two events were detected in the left upper parathyroid, three in the left lower parathyroid, two in the right upper parathyroid, and two in the pancreas. Of these events, we identified three indels and six nonsynonymous SNVs. A summary of the somatic variants identified in each tumor are shown in Table 3. Nine somatic variants, which were not present in the leukocyte samples, and, therefore, were not germline. Two somatic functional mutations outside the MEN1 gene were identified in the pancreatic insulinoma by WES. No somatic MEN1 gene mutation was found in any of the four tumors.

\section{Discussion and conclusions}

Genome-wide investigation of tumor and blood samples using WES and SNP array detected a novel MEN1 germline mutation (first hit) and tumor somatic mutations (second hit), confirming the two-hit model for tumor-suppressor genes. The heterogeneity between tumors (LOH in parathyroid tumors and no $\mathrm{LOH}$ in the pancreatic tumor) within a single patient suggests polyclonal tumorigenesis related to a predisposition arising from a single germline mutation.

The novel MEN1 germline mutation (c.1382_1383delAG; p.E462GfsX69) discovered in the present study was discovered by WES and confirmed by Sanger sequencing. This mutation was found in all four tumor samples and the blood sample and has not been reported in dbSNP135, the 1000 Genome Project, or the Korean control exome dataset. The brother and son of the proband did not harbor this mutation. The present study contributes to medical science by reporting a novel MEN1 germline mutation causing MEN1 syndrome and supports the clinical applicability of using WES to discover a causative mutation in the proband and to perform genetic testing on family members.

The $\mathrm{LOH}$ analysis detected the presence of chromosome 11 (where the MEN1 gene resides) LOH with chromosomal loss in all three parathyroid tumors. These findings demonstrate that the MEN1 germline mutation (first hit) and the chromosome $11 \mathrm{LOH}$ (second hit) caused biallelic inactivation of the tumor suppressor MEN1 gene, which drives tumorigenesis of these tumors (Fig. 3). Additional chromosomal abnormalities were detected in the left lower part of the parathyroid tumor, suggesting the occurrence of possible genome instability and passenger mutations, as reported previously in similar studies on parathyroid tumors $[14,15]$. No sign of $\mathrm{LOH}$ was found in the pancreatic tumor sample, suggesting that the mechanism of a MEN1 pancreatic tumor may be different from that of a MEN1 parathyroid tumor. These findings suggest that polyclonal tumorigenesis is related to a predisposition arising from a single germline mutation in MEN1 syndrome.

The somatic mutation analysis using WES revealed several possible driver mutations (other than MEN1). Because the pancreatic tumor did not harbor the MEN1 inactivation mutation (e.g., chromosome $11 \mathrm{LOH}$ in the parathyroid tumors), we tried to determine the possible driver mutations involved in tumorigenesis in addition to the haploinsufficiency of the germline MEN1 mutation. One stop-gain mutation in the ZNF429 gene and one frameshift mutation in the SCARF2 gene were discovered in the pancreatic tumor sample. These mutations may make a contribution to the tumorigenesis that is in addition to that made by the predisposition arising from the germline MEN1 mutation. However, these somatic mutations from the parathyroid and pancreatic tumors may simply be passenger mutations resulting from tumorigenesis. It is difficult to distinguish a driver mutation from a passenger mutation in tumor samples using the WES approach.

We discussed a WES base analytical pipeline that detected germline and somatic variations by performing variant calling using two algorithms (GATK and SAMtools) to assist in identifying true-positive variant calls. To increase the validation rate, SNVs and indels were filtered with a mapping quality score $>58$ in GATK and SAMtools. According to the SNP quality (SNPQ) and strand bias $(\mathrm{SB})$ analyses, the variants were filtered with SNPQ $\geq 300, \mathrm{SB}<-10$ in GATK and SNPQ $\geq 40$, and $20 \leq \mathrm{AF} \%<80$ in SAMtools. These parameters are based on a previous study [16].

Here, we compared the WES platform results, which use depth of coverage and alternative allele frequencies from mapped sequenced reads to estimate $\mathrm{LOH}$, with those of the SNP genotyping array. In the WES analysis, homozygous alleles were observed in chromosome 11 in all three parathyroid tumor samples, indicating $\mathrm{LOH}$. Array Comparative Genomic Hybridization (aCGH) and SNP genotyping arrays have been widely used as standard methods to detect copy-number variation and $\mathrm{LOH}$. However, with the rapid increase in genomic and exomic sequencing, LOH can be detected by WES, extending the ability of this powerful approach [17]. The resolution of these methods is limited by depth of coverage and the capture probe design. These data suggest that a WESbased platform is sufficient for identifying the genetic causes of various diseases in a diagnostic analysis and can be used to estimate germline and somatic mutations, as well as LOH simultaneously.

NGS allows characterization of genetic diseases with high accuracy and low cost. In particular, WES is an alternative approach to discovering variants that offers high 


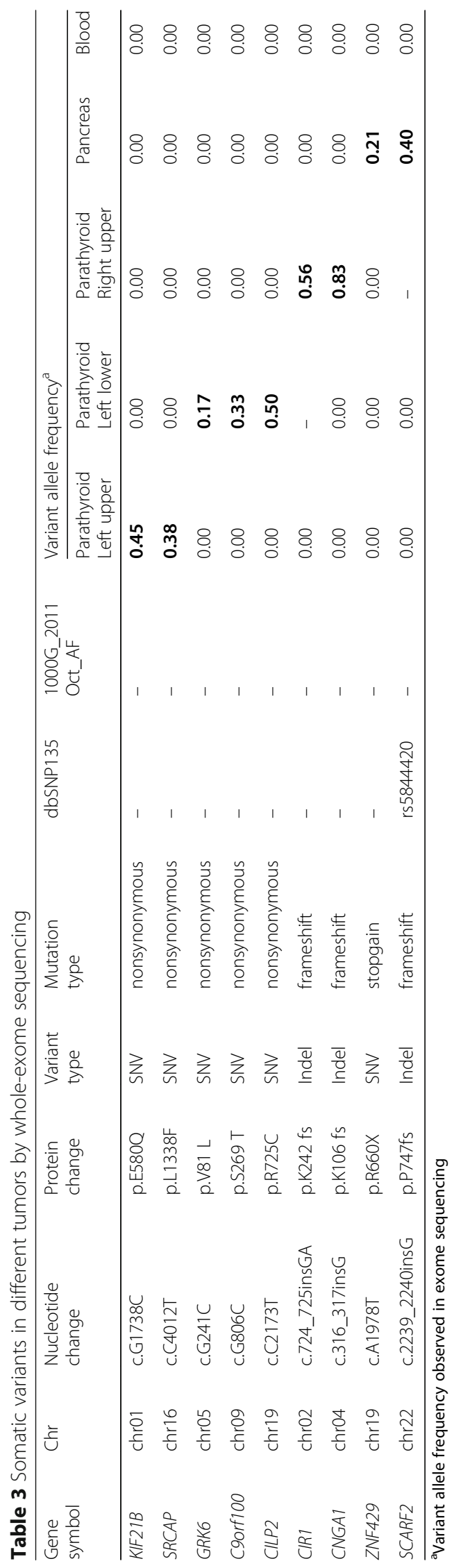




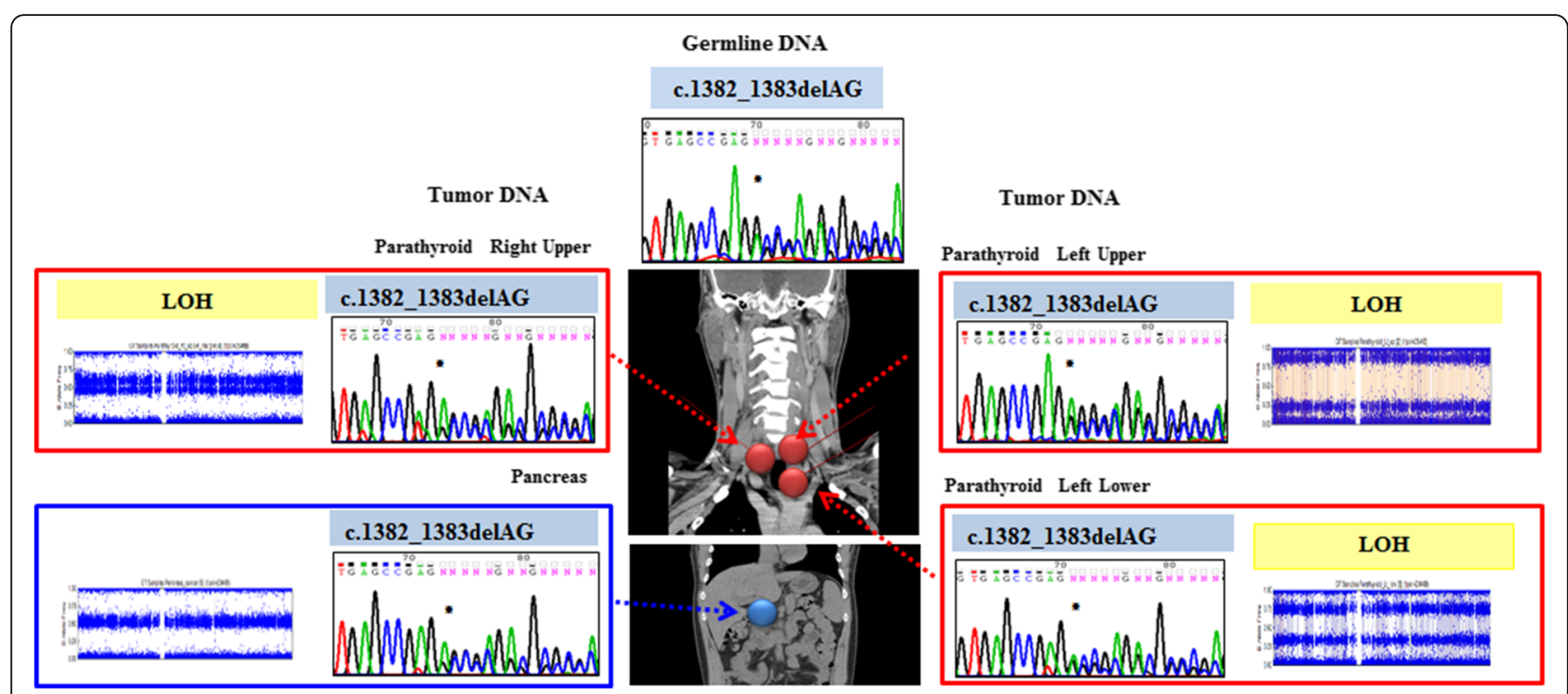

Fig. 3 Analysis of multiple endocrine tumors from the same patient. Computed tomography scans and the various nodules present on both the parathyroid glands (red arrow) and pancreas (blue arrow) of the same patient are shown. Each nodule shows the germline defect (blue). A second alteration (yellow) differed between the parathyroid gland and pancreas

specificity and sensitivity [18] and can be used to identify causative mutations in a number of Mendelian disorders, including monogenic diseases, as most causative mutations are on protein-coding regions. It also has the potential to identify de novo genetic variations through the WES platform. For these reasons, WES could be a surrogate to discover medically relevant genomic variations and diagnose genetic diseases at a genome-wide scale.

We identified a novel MEN1 germline mutation and tumor-specific somatic variants in a patient with MEN1 and multiple parathyroid and pancreatic tumors. Heterogeneity between tumors within a single patient suggests polyclonal tumorigenesis related to a predisposition arising from a single germline mutation. The present study demonstrates the clinical applicability of the WES strategy, which uses multiple tumor samples and a blood sample, to investigations of disease pathogenesis and evaluations of clinical genetics for patients and family members.

\section{Additional files}

Additional file 1: Table S1. Number of somatic variants observed in four tumor types. (DOC $64 \mathrm{~kb})$

Additional file 2: Figure S1. Analysis of loss of heterozygosity (LOH) by single-nucleotide polymorphism (SNP) arrays. (A) Chromosomal alteration (gain, loss, or loss of heterozygosity) in parathyroid and pancreatic tumors. $\mathrm{LOH}$ of 11 was detected in the parathyroid tumor. $\mathrm{LOH}$ can easily be observed using a $\mathrm{B}$ allele frequency (BAF) plot by noting the absence of heterozygotes. (B) Chromosome 11 from parathyroid and pancreas. The upper part of the panel shows genotypes of the SNPs expressed as the BAF. The lower part of the panel shows the DNA copy number expressed on a base-2 log scale (log ratio), and the red line corresponds to two copies of DNA. (TIFF $789 \mathrm{~kb})$

\section{Abbreviations}

aCGH: Array comparative genomic hybridization; AF: Allele frequency; BAF: B allele frequency; CT: Computed tomography; GATK: Genome Analysis Toolkit; KRG: Korean Reference Genome; LogR: Log2 intensity ratio; LOH: Loss of heterozygosity; MEN1: Multiple endocrine neoplasia type 1; NGS: Next-generation sequencing; SAMtools: Sequence Alignment Map tools; SB: Strand bias; SIFT: Sorting Intolerant Form Tolerant;

SNPQ: Single nucleotide polymorphism quality; SNVs: Single nucleotide variants; WES: Whole-exome sequencing

\section{Acknowledgments}

The specimens used in this study were provided by Chungbuk National University Hospital, a member of the National Biobank of Korea, which is supported by the Ministry of Health, Welfare, and Family Affairs. All samples derived from the National Biobank of Korea were obtained with informed consent under institutional review board approved protocols.

\section{Funding}

This study was supported by the Korea National Institute of Health intramural research grant 4800-4861-312-210 (no. 2016-NG61003-00).

Availability of data and materials

All data generated or analyzed during this study are included in this article.

Authors' contributions

BYK performed the data analysis and prepared the manuscript. MHP supervised the data analysis and prepared the manuscript. HMW collected the whole-exome data. HYJ advised on the data analysis. JHK collected the phenotype data. HJC supervised the project, prepared the manuscript, and advised on the data analysis. SKK supervised the project, prepared the manuscript, and advised on the data analysis. All authors read and approved the final manuscript.

Ethics approval and consent to participate

The Institutional Review Board of Chungbuk National University Hospital and Korea National Institutes of Health $(\mathrm{NIH})$ approved this study. All of participants were informed to participate in this study with written consents.

Consent for publication

Written informed consent were obtained from the patient for the publication of their clinical details and images. 


\section{Competing interests}

The authors declare that they have no competing interests.

\section{Publisher's Note}

Springer Nature remains neutral with regard to jurisdictional claims in published maps and institutional affiliations.

\section{Author details}

${ }^{1}$ Division of Intractable Diseases, Center for Biomedical Sciences, Korea National Institute of Health, 187 Osongsaengmyeing2-ro, Cheongju-si, Chungcheongbuk-do 28159, South Korea. ${ }^{2}$ Department of Anatomy, Department of Biomedical Science, Neuroscience Research Institute, Seoul National University College of Medicine, 28 Yongon-dong, Chongno-gu, Seoul, South Korea. ${ }^{3}$ Department of Surgery, Eulji University Hospital,

Daejeon, South Korea.

Received: 28 December 2016 Accepted: 11 September 2017

Published online: 02 October 2017

\section{References}

1. Hoff AO, Cote GJ, Gagel RF. Multiple endocrine neoplasias. Annu Rev Physiol. 2000;62:377-411

2. Thakker RV. Multiple endocrine neoplasia type 1 (MEN1). Best Pract Res Clin Endocrinol Metab. 2010;24:355-70.

3. Chandrasekharappa SC, Guru SC, Manickam P, Olufemi SE, Collins FS, Emmert-Buck MR, Debelenko LV, Zhuang Z, Lubensky IA, Liotta LA, Crabtree JS, Wang Y, Roe BA, Weisemann J, Boguski MS, Agarwal SK, Kester MB, Kim YS, Heppner C, Dong Q, Spiegel AM, Burns AL, Marx SJ. Positional cloning of the gene for multiple endocrine neoplasia-type 1. Science. 1997;276:404-7.

4. Kouvaraki MA, Lee JE, Shapiro SE, Gagel RF, Sherman SI, Sellin RV, et al. Genotype-phenotype analysis in multiple endocrine neoplasia type 1. Arch Surg. 2002;137:641-7.

5. Carling T. Multiple endocrine neoplasia syndrome: genetic basis for clinical management. Curr Opin Oncol. 2005;17:7-12.

6. Brandi ML, Gagel RF, Angeli A, et al. Guidelines for diagnosis and therapy of MEN type 1 and type 2. J Clin Endocrinol Metab. 2001;86:5658-71.

7. Wheeler DA, Srinivasan M, Egholm M, Shen $Y$, et al. The complete genome of an individual by massively parallel DNA sequencing. Nature. 2008;452:872-6.

8. Yang Y, Muzny DM, Reid JG, et al. Clinical whole-exome sequencing for the diagnosis of mendelian disorders. N Engl J Med. 2013;369(16):1502-11.

9. Taylor JC, Martin HC, Lise S, et al. Factors influencing success of clinical genome sequencing across a broad spectrum of disorders. Nat Genet. 2015:47:717-26.

10. Li H, Durbin R. Fast and accurate short read alignment with burrowswheeler transform. Bioinformatics. 2009:25:1754-60.

11. McKenna A, Hanna M, Banks E, Sivachenko A, Cibulskis K, Kernytsky A, Garimella K, Altshuler D, Gabriel S, Daly M, DePristo MA. The genome analysis toolkit: a MapReduce framework for analyzing next-generation DNA sequencing data. Genome Res. 2010;20:1297-303.

12. $\mathrm{Ng}$ PC, Henikoff S. SIFT: predicting amino acid changes that affect protein function. Nucleic Acids Res. 2003;31(13):3812-4.

13. Adzhubei IA, Schmidt S, Peshkin L, Ramensky VE, Gerasimova A, Bork P, Kondrashov AS, Sunyaev SR. A method and server for predicting damaging missense mutations. Nat Methods. 2010;7:248-9.

14. Newey PJ, Nesbit MA, Rimmer AJ, Attar M, Head RT, Christie PT, Gorvin CM, Stechman M, Gregory L, Mihai R, et al. Whole-exome sequencing studies of nonhereditary (sporadic) parathyroid adenomas. J Clin Endocrinol Metab. 2012:97(10):E1995-2005.

15. Cromer MK, Starker LF, Choi M, Udelsman R, Nelson-Williams C, Lifton RP, Carling T. Identification of somatic mutations in parathyroid tumors using whole-exome sequencing. J Clin Endocrinol Metab. 2012;97(9):E1774-81.

16. Park MH, Rhee H, Park JH, Woo HM, Choi BO, Kim BY, Chung KW, Cho YB, Kim HJ, Jung JW, Koo SK. Comprehensive analysis to improve the validation rate for single nucleotide variants detected by next-generation sequencing. PLoS One. 2014;9(1):e86664.

17. Sathirapongsasuti $J H$, Lee $H$, Hors BAJ, et al. Exome sequencing-based copynumber variation and loss of heterozygosity detection: exome CNV. Bioinformatics. 2011;27(19):2648-54.

18. Ng SB, Turner EH, Robertson PD, Flyqare SD, et al. Targeted capture and massively parallel sequencing of 12 human exomes. Nature. 2009;461:272-6.

\section{Submit your next manuscript to BioMed Central and we will help you at every step:}

- We accept pre-submission inquiries

- Our selector tool helps you to find the most relevant journal

- We provide round the clock customer support

- Convenient online submission

- Thorough peer review

- Inclusion in PubMed and all major indexing services

- Maximum visibility for your research

Submit your manuscript at www.biomedcentral.com/submit 